\title{
Análisis osteológico de la saraca Brevoortia aurea (Spix) (Actinopterygii: Clupeidae) en el Atlántico suroccidental
}

\author{
Osteological analysis of the menhaden Brevoortia aurea (Spix) \\ (Actinopterygii: Clupeidae) in the southwestern Atlantic \\ Valeria Segura' ${ }^{1}$ y Juan M. Díaz de Astarloa ${ }^{1,2}$ \\ ${ }^{1}$ Departamento de Ciencias Marinas, Facultad de Ciencias Exactas y Naturales, Universidad Nacional de Mar del Plata, \\ Funes 3350, B7602AYL, Mar del Plata, Argentina \\ ${ }^{2}$ Consejo Nacional de Investigaciones Científicas y Técnicas (CONICET). Instituto Nacional de Investigación y Desarrollo Pesquero \\ (INIDEP), Paseo Victoria Ocampo 1, B7602HSA, Mar del Plata, Argentina \\ vsegura@mdp.edu.ar
}

\begin{abstract}
Resumen.- Se efectuó un análisis osteológico comparativo entre especímenes de Brevoortia aurea procedentes de dos regiones del Atlántico suroccidental. El objetivo fue describir la osteología de la saraca con el fin de aportar características diagnósticas que permitan la discriminación de la especie con otras especies nominales del género. Se utilizaron técnicas de desarticulación en fresco para ejemplares grandes (162 a 285 mm de largo estándar) y de clareado y tinción para individuos pequeños (31,5 a 112,4 mm de largo estándar). El análisis detallado de las estructuras óseas permitió la discusión de las afinidades y diferencias con otras especies de clupeidos.
\end{abstract}

Palabras clave: Peces, taxonomía, osteología

\begin{abstract}
A comparative osteological analysis between specimens of Brevoortia aurea from two regions of the southwestern Atlantic was done. The aim of this paper was to describe the osteology of the menhaden in order to bring diagnostic characteristics that allow species discrimination from other congeneric species. Methods of preparing disarticulated skeletons of large specimens (162-285 mm SL), and cleared and stained techniques for small specimens (31.5$112.4 \mathrm{~mm}$ SL) were used. The detailed osteological analysis allowed the discussion of the similarities and differences among other clupeid species.
\end{abstract}

Key Words: Fishes, taxonomy, osteology

\section{Introducción}

El género Brevoortia Gill se distribuye en el Atlántico occidental desde Nueva Escocia $\left(45^{\circ} \mathrm{N}\right)$ hasta Argentina $\left(40^{\circ} \mathrm{S}\right)$. Según Whitehead (1985) dos especies nominales del género estarían presentes en el Atlántico suroccidental: Brevoortia aurea (Spix, 1829) que se extendería desde Bahía, Brasil, hasta la boca del Río de la Plata, y B. pectinata (Jenyns, 1842) que ocurriría desde Río Grande do Sul, Brasil hasta Bahía Blanca, Argentina (Hildebrand 1948).

A partir del trabajo de Hildebrand (1948) algunos autores reconocen a las dos especies nominales como válidas (De Buen 1950, Ringuelet \& Arámburu 1960, Ringuelet et al. 1967, Figueiredo \& Menezes 1978, Menni et al. 1984, Menezes et al. 2003). Otros autores mencionan la existencia de una sola especie, que por prioridad correspondería a Brevoortia aurea (Reintjes 1969, Hormaechea 1983, Cassia \& García de la Rosa
1993, Cousseau \& Díaz de Astarloa 1993, Cousseau \& Denegri 1997, Cousseau et al. 1998). Un tercer grupo de autores considera que deberían aceptarse las conclusiones de Hildebrand (1948) sobre la presencia de las dos especies hasta que un mayor número de ejemplares fuera adecuadamente estudiado (Berry 1964, Whitehead 1985, Carvalho-Filho 1999).

Cousseau \& Díaz de Astarloa (1993) analizan estadísticamente 489 ejemplares de Brevoortia procedentes del sur de Brasil (Rio Grande) hasta Bahía Blanca (Argentina), incluyendo el área de superposición que Whitehead (1985) señala para las dos especies nominales, esto es la boca del Río de la Plata. La comparación de los caracteres morfométricos y merísticos observados, con los dados por otros autores indica que no se podrían distinguir las dos especies sobre la base de los caracteres que se toman normalmente en consideración. Los autores hacen una aclaración con respecto al número de hileras de escamas 
que cruza el flanco del cuerpo y que Hildebrand (1948) establece como el mejor carácter para distinguir a $B$. pectinata de $B$. aurea. Cousseau \& Díaz de Astarloa (1993) comprobaron que dicho número es proporcional al tamaño de los ejemplares. Finalmente, los autores concluyen que en la región entre Rio Grande do Sul y Bahía Blanca $\left(32^{\circ} \mathrm{S}\right.$ y $\left.41^{\circ} \mathrm{S}\right)$ sólo se encuentra una sola especie, Brevoortia aurea.

Cassia \& García de la Rosa (1993) describen y comparan diferentes estadios de desarrollo de larvas y postlarvas de Brevoortia aurea del norte de Argentina con muestras procedentes del sur de Brasil descriptas con el nombre de $B$. pectinata, y concluyen que ambas especies nominales representan variaciones latitudinales de una sola especie, $B$. aurea.

El objetivo del presente trabajo fue describir la osteología de ejemplares de Brevoortia aurea con el fin de aportar características diagnósticas que permitan la discriminación de la especie con otras especies nominales que pudieran estar presentes en el Atlántico suroccidental.

\section{Materiales y métodos}

Tomando en cuenta los antecedentes sobre la identificación de especies de Brevoortia en el área de estudio del presente trabajo, y de acuerdo a las características merísticas y morfométricas obtenidas de los ejemplares objeto de este estudio, el material analizado corresponde a la especie nominal Brevoortia aurea.

Se analizó un total de 49 ejemplares, de los cuales 10, de tallas entre 53,6 y 112,4 mm de largo estándar procedieron de la laguna Los Patos (30³0' a 32 $12^{\circ}$ 'S y $50^{\circ}$ a $51^{\circ} \mathrm{W}$ ) (Brasil), mientras que el resto de los ejemplares, de largo estándar de entre 31,5 y $285 \mathrm{~mm}$, provinieron de la laguna Mar Chiquita (37²32' a

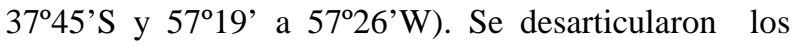
esqueletos y neurocráneos para individualizar cada uno de los huesos. Se siguió la metodología propuesta por Díaz de Astarloa (1994) para ejemplares adultos o bien el método de Mayden \& Wiley (1984) para juveniles. Se utilizaron técnicas de clareado y tinción para los peces de pequeño tamaño. En este trabajo se siguieron las indicaciones formuladas por Wasserssug (1976) y Kawamura \& Hosoya (1991). Se confeccionaron dibujos de los huesos en tamaño original y también utilizando una lupa estereoscópica provista de cámara clara. En el caso particular de los arcos branquiales, las branquispinas fueron removidas y no fueron dibujadas.
Para la nomenclatura de los mismos se siguió en líneas generales a Cervigón (1980) y en casos particulares a Rojo (1988) y Patterson \& Johnson (1995).

\section{Resultados}

\section{Neurocráneo}

Presenta las tres características típicas del superorden Clupeomorpha: los forámenes temporales (Ft) delimitadas por los frontales y los parietales (Fig. 1C); las fosas preepióticas (F.p) limitados por el parietal, pterótico y epiótico (Fig. 1C); y las fenestras auditivas (F.a) rodeadas por los proóticos, exoccipitales y basioccipital (Fig. 1B).

\section{Región etmoesfenoidal}

Constituida por el etmoides (Etm) y el prevómer (Pv) como huesos impares y los etmoides laterales (E.l) y nasales (Na) como huesos pares.

El etmoides en vista dorsal presenta un componente rostral (E.r) en forma de escudo con dos procesos laterales dirigidos posteriormente y con una escotadura en la región anterior (Fig. 1A). En la región anterior se expande en dos procesos laterales formando los bordes frontal y lateral del rostro. El etmoides tiene una escotadura posterior que forma el límite anterior de la fontanela etmoidal (Fig. 1A). A la superficie dorsal, y extendiéndose a cada lado del hueso, están adosados los etmoides laterales, de forma alar y laminares (Fig. 1A y C). Por debajo del etmoides, se extiende el prevómer, sin dientes y delgado, el cual se ensancha hacia la parte anterior (Fig. 1B). Hacia el borde lateral del etmoides se sitúan los nasales, huesos de canal pequeño, que se encuentran ubicados inmediatamente por debajo de la piel (Fig. 2).

\section{Región orbitotemporal}

Esta región está constituida por los frontales (F), parietales (Par), pteroesfenoides (Pt) y circumorbitarios como huesos pares y el basiesfenoides (Bs) y el orbitoesfenoides (Orb) como huesos impares.

Los frontales son huesos que están alargados en el eje horizontal. Anteriormente se separan limitando la fontanela etmoidal (Fe), mientras que están unidos hacia la parte posterior por una sutura sigmoidea (Fig. $1 \mathrm{~A}$ y C). Se encuentran recorridos lateralmente por el canal sensorial supraorbitario (Css) (Fig. 1C). Por detrás de los frontales se encuentran los parietales, de 
A

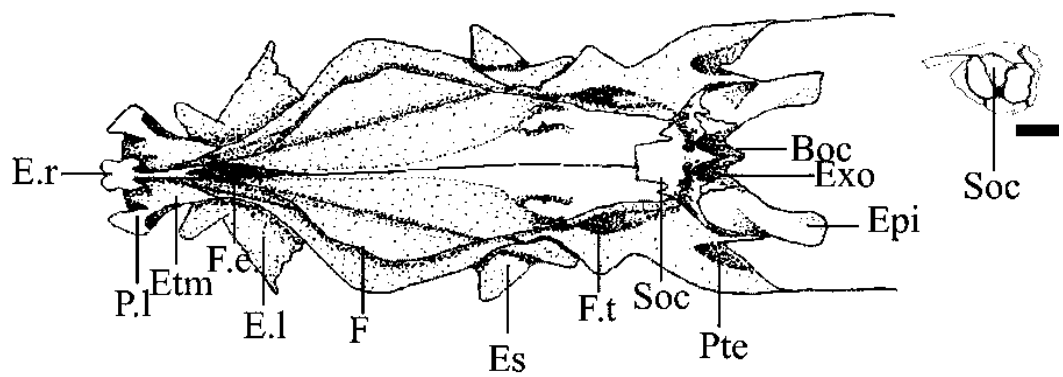

B

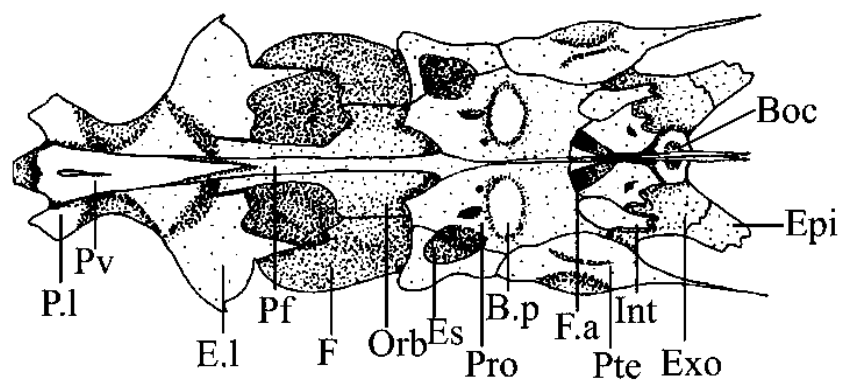

C

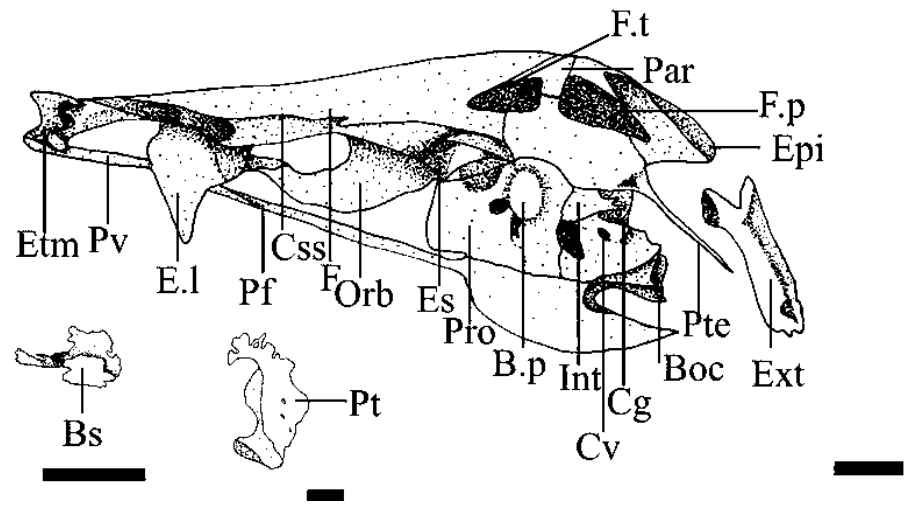

Figura 1

Boc: basioccipital

Cv: canal del nervio vago

Epi: epiótico

Ext: extraescapular

F.p: fosa preepiótica

Par: parietal

Pt: pterosfenoides

Escalas: 5 mm

Boc: basioccipital

Cv: vagal nerve canal

Epi: epiotic

Ext: extrascapular

F.p: preepiotic fossa

Par: parietal

Pt: pterosphenoid

Scale bars: $5 \mathrm{~mm}$
Neurocráneo de $B$.

Css: canal sensorial supraorbitario

E.r: escudo rostral

F: frontal

F.t: foramen temporal

Pf: paraesfenoides

Pte: pterótico

Bs: basiesfenoides
E.l: etmoides lateral
Es: esfenótico
F.a: fenestra auditiva
Int: intercalar
P.l: proceso lateral
P.v: prevomer

Bs: basiesfenoides

E.l: etmoides latera

F.a: fenestra auditiva

P.l: proceso latera
Cg: canal del nervio glosofaríngeo

Etm: etmoides

Exo: exoccipital

F.e: fontanela etmoidal

Orb: orbitoesfenoides

Pro: proótico

Soc: supraoccipital

Neurocranium of B. aurea. A: dorsal; B: ventral; C: lateral views

B.p: prootic bulla

Css: supraorbitary sense canal

E.r: escudo rostral

F: frontal

F.t: temporal foramen

Pf: parasphenoid

Pte: pterotic
Bs: basisphenoid

E.l: lateral ethmoid

Es: sphenotic

F.a: otical fenestra

Int: intercalar

P.l: lateral process

P.v: prevomer
Cg: glossopharyngeal nerve canal Etm: ethmoid

Exo: exoccipital

F.e: ethmoidal fontanella

Orb: orbitosphenoid

Pro: prootic

Soc: supraoccipital 


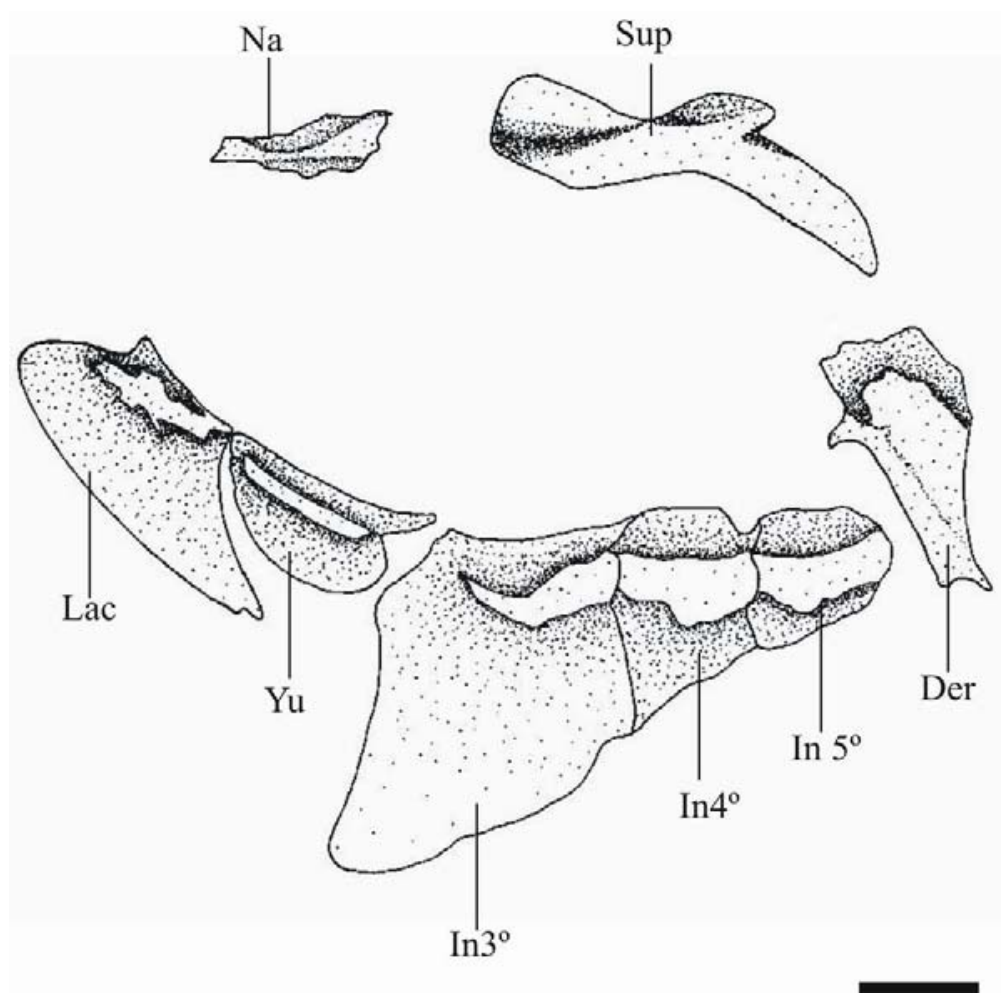

Figura 2

Huesos circumorbitarios y nasal de B. aurea. Der: dermoesfenotico; In $3^{\circ}, \operatorname{In} 4^{\circ}, \operatorname{In} 5^{\circ}$ : huesos infraorbitarios tercero, cuarto y quinto, respectivamente; Lac: lacrimal; Na: nasal; Sup: supraorbitario; Yu: yugal. Cara externa izquierda, vista lateral.

Escala: 5 mm

Circumorbital and nasal bones of B. aurea. Der: dermosphenotic; $\operatorname{In} 3^{\circ}$, $\operatorname{In} 4^{\circ}$, In $5^{\circ}$ : Third, fourth and fifth, infraorbital bones respectively; Lac: lacrymal; Na: nasal; Sup: supraorbital; Yu: Jugal. Left outer side, lateral view. Scale bar: 5 mm

pequeño tamaño, presentando forma de $\mathrm{L}$ invertida y acostada, con el vértice adosado a las expansiones laterales del supraoccipital (Fig. 1C).

El basiesfenoides (Bs) es un hueso muy pequeño, extendido anteriormente en un largo y angosto proceso. Posteriormente presenta un par de cóndilos laterales que están articulados con los pteroesfenoides (Fig. 1C). Los pterosfenoides son de forma irregular (Fig. 1C) y se unen anteriormente al borde posterodorsal del orbitoesfenoides por medio de un cartílago. El orbitoesfenoides (Fig. 1B y C) también presenta forma irregular con cóndilos ubicados en la región posterosuperior del mismo que articulan con los frontales, mientras que en la región anterior presenta un proceso corto y curvado dorsalmente que se une a los dos etmoides laterales (Fig. 1C).
La serie circumorbitaria está formada por el lacrimal (Lac), el yugal $(\mathrm{Yu})$, al cual le siguen el $3^{\circ}, 4^{\circ}$ y $5^{\circ}$ circumorbitarios $\left(\operatorname{In} 3^{\circ}-5^{\circ}\right)$. A continuación está el dermoesfenótico (Der) por encima el supraorbitario (Sup), el cual cierra la órbita en la región superior. Todos estos huesos presentan en sus superficies un pliegue óseo, el cual forma un canal para el paso de la rama laterosensorial circumorbitaria (Fig. 2).

\section{Región oticooccipital}

Formada por los huesos pterótico (Pte), proótico (Pro), epiótico (Epi), intercalar (Int), esfenótico (Es), exoccipital (Exo), supraoccipital (Soc), y extraescapular (Ext). Excepto el supraoccipital, todos los huesos de la región son pares. 
El pterótico en la región anterior es robusto y está articulado con la rama vertical del parietal y posteriormente a la rama inferior del epiótico (Fig. 1C). Hacia la región posterior se angosta abruptamente terminando en un proceso largo en forma de espina (Fig. 1B). El proótico está constituido básicamente por la bulla ótica (B.p) de gran tamaño y forma ovalada (Fig. 1B y C). Por detrás del mismo, se encuentra el intercalar de pequeño tamaño, ovalado anteriormente y de forma rectangular posteriormente (Fig. 1B y C). Ventral y posterior al intercalar se encuentra el exoccipital que presenta en su parte posterior dos orificios: uno más grande que delimita el canal del nervio vago $(\mathrm{Cv})$ y otro menor para el paso del nervio glosofaríngeo (Cg) (Fig. 1B y C). Junto con el basioccipital forman gran parte de la cámara que aloja a los otolitos.

El esfenótico presenta forma ovalada en su región dorsal siendo irregular su región inferior. Forma con el proótico una acusada concavidad donde se adosa el cóndilo anterior del hiomandibular (Figs. 1B y C).

El supraoccipital cierra posterodorsalmente el neurocráneo; hacia adelante articula con el frontal. Se hace robusto hacia la región posterior donde articula con el epiótico (Fig. 1A). El epiótico es un hueso de forma más o menos triangular que cierra la caja craneana posterolateralmente (Figs. 1A, B y C).

El extraescapular tiene forma de varilla alargada en el eje vertical y está dividida en el extremo dorsal en dos procesos cortos (Fig. 1C).

\section{Región basicraneal}

Está formada por dos huesos impares: el basioccipital (Boc) y el paraesfenoides (Pf). El primero articula anteriormente con el paraesfenoides y hacia atrás con la primera vértebra (Fig. 1B y C). El paraesfenoides es un hueso alargado que se estrecha hacia la región anterior en donde encaja el prevómer y se extiende posteriormente presentando expansiones alares (Fig. 1B y C). El mismo forma parte del paladar.

\section{Branquiocráneo}

\section{Región oromandibular}

Formada por el premaxilar (Pm), maxilar (M), dos supramaxilares (Sm), el dentario (Dent), angular (Ang), retroarticular (Ret) y el coronomeckeliano (Cor). Todos son huesos pares.
El premaxilar está situado por debajo del etmoides, es muy pequeño, presenta una forma más o menos triangular y está desprovisto de dientes al igual que el maxilar, el cual, presenta un cóndilo relativamente grande en su región anterosuperior, que articula con el borde superior de la cabeza del prevómer. Posteriormente tiene otro cóndilo menos definido y más pequeño, llamado cóndilo palatino que articula con el palatino. Detrás del mismo están situados los dos supramaxilares que se caracterizan por ser laminares (Fig. 3A).

El dentario es un hueso sin dientes, alargado, con una cavidad en la porción posterior donde encaja el angular. El angular en su parte media dorsal; presenta un proceso ascendente terminado en punta, que encaja con la porción posterodorsal del dentario (Fig. 3A). El retroarticular se encuentra firmemente unido a la parte posterior del angular (Fig. 3B). Es un hueso alargado en el eje horizontal. En la cara interna del angular se encuentra insertado el coronomeckeliano de tamaño muy pequeño y de forma irregular (Fig. 3B).

\section{Arco palatocuadrado o suspensorio}

Está formado por el cuadrado (Cu), simpléctico (Sim), ectopterigoides (Ect), endopterigoides (End), metapterigoides (Met), palatino (Pal) e hiomandibular (Hio). Todos ellos son huesos pares.

En Brevoortia, a diferencia de la mayoría de los peces en los cuales el cuadrado tiene una forma triangular típica, presenta forma de L. En el vértice inferior presenta un cóndilo que articula con el angular. Por debajo de la rama horizontal del cuadrado, separado por cartílago, se encuentra el simpléctico con forma de varilla (Fig. 3A).

El ectopterigoides es un hueso alargado en el eje vertical, se encuentra superpuesto a la cara interna de la rama vertical del cuadrado. Dorsalmente está articulado al palatino y endopterigoides y dorsoposteriormente al metapterigoides. El endopterigoides es redondeado en su cara dorsal. Anteriormente articula con el palatino, y posteriormente con el metapterigoides mediante una articulación en forma de S. Ventralmente se relaciona con el ectopterigoides. El palatino es un hueso triangular, no lleva dientes y está articulado por su parte anterior con el maxilar, posteriormente con el endopterigoides, y ventroposteriormente con el ectopterigoides (Fig. 3A).

El hiomandibular es un hueso prominente, con dos cóndilos dorsales para la articulación con el 
A

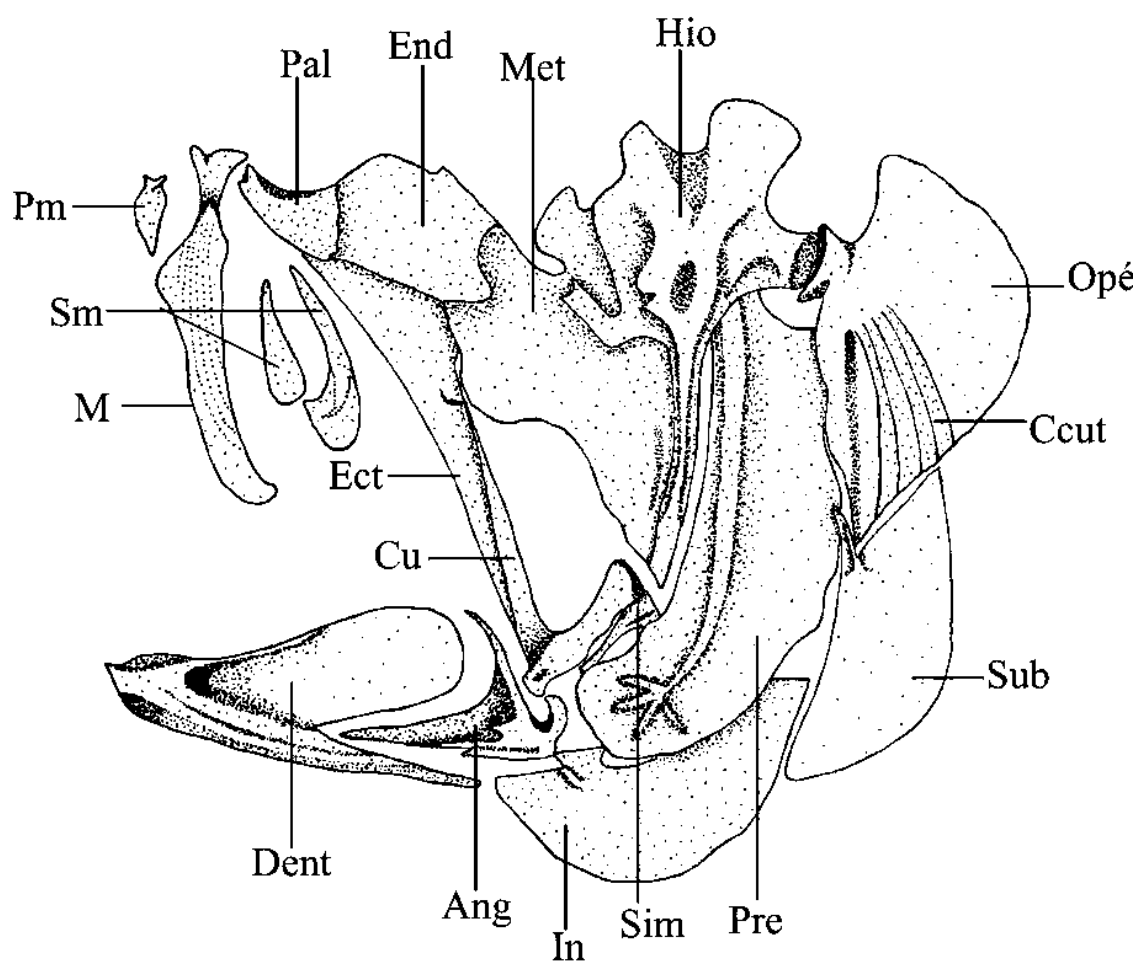

B
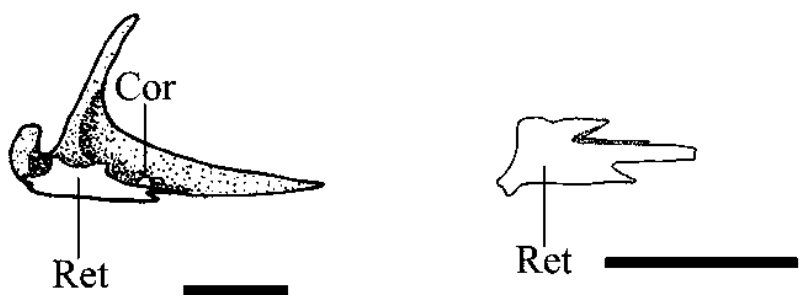

Figura 3

Braquiocráneo de $B$. aurea.

A: Región oromandibular, arco palatocuadrado o suspensorio y serie opercular. Cara externa, lado izquierdo. B: Cara interna del angular izquierdo. Ang: angular; Ccut: canales cutáneos radiales; Cor: coronomeckeliano; Cu: cuadrado; Dent: dentario;

Ect: ectopterigoides; End: endopterigoides; Hio: hiomandibular; In: interopérculo; M: maxilar; Met: metapterigoides;

Pal: palatino; Pre: preopérculo; Pm: premaxilar; Opé: opérculo; Ret: retroarticular; Sim: simpléctico; Sub: subopérculo;

Sm: supramaxilares. Escalas: 5 mm

Brachiocranium of $B$. aurea.

A: Oromandibular region, palatoquadrate arch and opercular series. Left outer side. B: Inner side of left angular. Ang: anguloarticular;

Ccut: cutaneous radial canals; Cor: coronomeckelian; Cu: quadrate; Dent: dentary; Ect: ectopterygoid; End: endopterygoid; Hio: hyomandibular; Inte: interopercular; M: maxilla; Met: metapterygoid; Pal: palatine; Pre: preopercular; Pm: premaxilla;

Opé: opercular; Ret: retroarticular; Sim: symplectic; Sub: subopercular; Sm: supramaxillar. Scale bars: 5 mm 
neurocráneo a través del esfenótico y pterótico y el cóndilo posterolateral que articula con el opérculo. Se encuentra alargado en el eje vertical. Una proyección anterior y de textura laminar se adosa a la cara externa del metapterigoides (Fig. 3A).

\section{Serie opercular}

Constituida por los siguientes huesos: el preopérculo (Pre), opérculo (Opé), subopérculo (Sub) e interopérculo (In). El preopérculo es un hueso alargado y presenta un canal central que en su región ventral se ramifica en pequeños canales radiales. El opérculo es un hueso prominente, con canales cutáneos radiales (Ccut), su borde posterior es redondeado y anterodorsalmente presenta una concavidad que articula con el cóndilo posterior del hiomandibular. El subopérculo tiene una escotadura en $\mathrm{V}$ donde se adosa la parte ventral del opérculo. Por su parte anterior se relaciona con el preopérculo y con el interopérculo. El interopérculo tiene forma de medialuna. Está unido al retroarticular por medio de un ligamento y articula dorsalmente con el preopérculo y con el subopérculo. (Fig. 3A).

\section{Región hioidea}

Está formada por el urohial y el basihial (Ba) como huesos impares, y por los huesos hipohial superior (Hsup), hipohial inferior (Hinf), ceratohial (Cer), epihial (Epi), interhial (Inte) y los radios branquiostegos (R.B), como elementos pares.

El urohial es un hueso alargado, que presenta un ensanchamiento anterior que termina en punta. Posteriormente presenta unas prolongaciones laterales en forma de alas (Fig. 4B). Anteriormente al mismo se encuentra el basihial sin dientes que tiene gran parte de su superficie cartilaginosa siendo su porción ósea muy reducida y laminar. (Fig. 4A).

Los hipohiales son huesos de pequeño tamaño y presentan forma triangular (Fig. 4A).

El ceratohial es un hueso alargado con sus extremos anterior y posterior redondeados. Presenta un foramen u orifico en la región posterior que probablemente sea el paso de la arteria hioidea (Rojo 1988). En la región inferior presenta cuatro pequeños orificios donde están insertos los primeros cuatro radios branquióstegos. Hay un total de 7 radios branquióstegos, los cuales son laminares y posteriormente de mayor tamaño y grosor. Branquióstegos I-V están unidos al ceratohial, mientras que el VI se adosa al ceratohial y al epihial y el VII está adherido al epihial. El epihial es de forma triangular y presenta gran parte de su superficie perforada. Está unido al hiomandibular por medio del interhial, el cual es muy pequeño y con forma de varilla (Fig. 4A).

\section{Arcos branquiales (Fig. 5A y B)}

Están constituidos por tres huesos impares, los basibranquiales (B1-B3), tres pares de hipobranquiales (H1-H3), cinco pares de ceratobranquiales (C1-C5), cuatro pares de epibranquiales (E1-E4), tres pares de infrafaringobranquiales (I1-I3) y dos pares de placas dentarias (Pld1 y PLd2).

Los basibranquiales están situados en la línea media de la cavidad bucal. El primero es pequeño y presenta forma triangular, mientras que los restantes son alargados y presentan un leve ensanchamiento en la región anterior. El tercero, a diferencia del segundo basibranquial, presenta en la región posterior una acanaladura donde encaja el hipobranquial tercero. (Fig. $5 A)$.

El primer par de hipobranquiales (H1) está formado por huesos largos y están ligeramente curvados dorsalmente en sus extremos. Los segundos hipobranquiales (H2) son más cortos que los primeros y los terceros (H3) son muy pequeños, con forma cónica. (Fig. 5A).

Los ceratobranquiales son los huesos más largos de la serie. Los cuartos ceratobranquiales presentan en la región media del hueso una quilla (Fig. 5A); se adelgazan anteriormente terminando en punta. Los quintos ceratobranquiales son huesos sin dientes delgados y curvados; en sus lados internos presentan expansiones laminares quebradizas. (Fig. 5A).

Los primeros tres pares de epibranquiales (E1-E3) son alargados, cada uno de ellos presenta en su cara interna una expansión delgada, quedando definido dos procesos de articulación en la región anterior de los mismos. El cuarto par de epibranquiales está sumamente modificado, tiene forma de hoja y constituye parte del órgano o bolsa epibranquial junto con las placas dentarias y el quinto arco branquial (Fig. $5 \mathrm{~A} \mathrm{y} \mathrm{B).} \mathrm{El} \mathrm{mismo} \mathrm{presenta} \mathrm{en} \mathrm{la} \mathrm{línea} \mathrm{media,} \mathrm{una}$ expansión cartilaginosa de consistencia débil y con su superficie perforada por pequeños orificios. Está unido lateralmente a las placas dentarias. Este par de epibranquiales carece de dientes (Fig. 5A y B).

El primer par de infrafaringobranquiales es triangular y de pequeño tamaño. Mantiene unido el aparato branquial al cráneo. Los infrafaringobranquiales

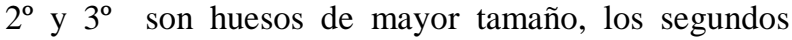


A

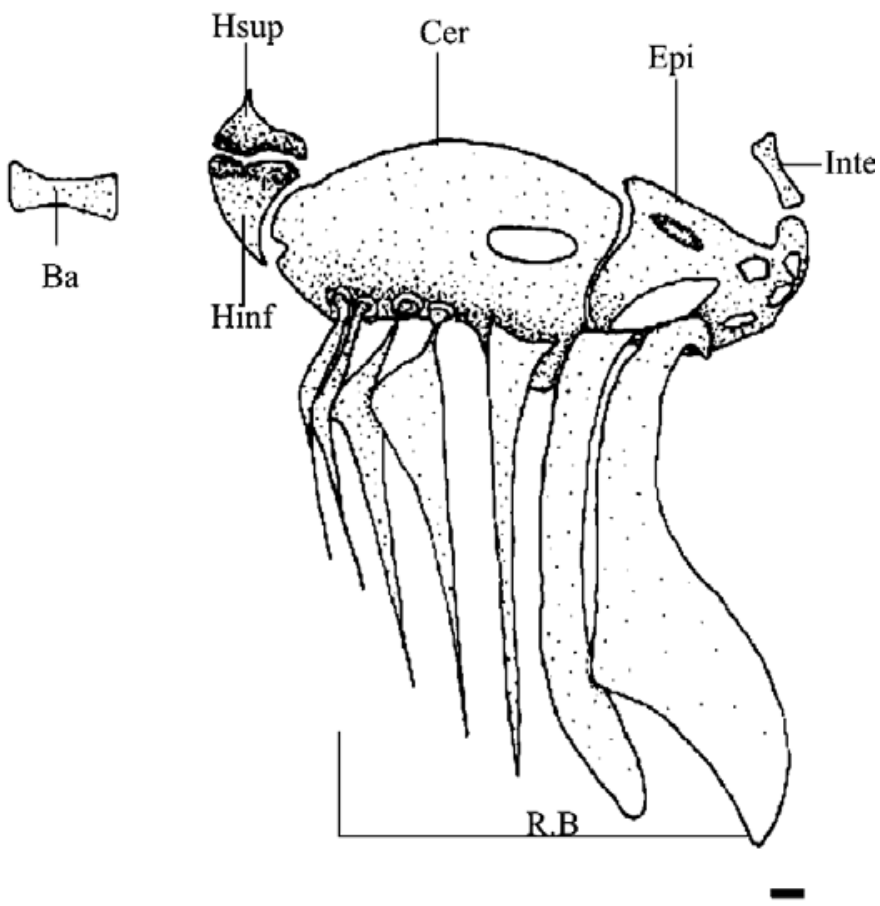

B

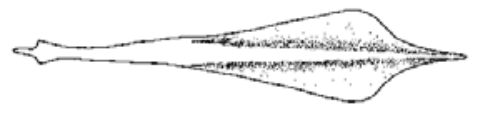

2

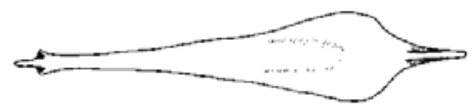

3

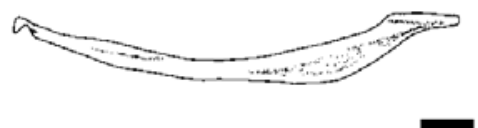

Figura 4

Región hioidea de B. aurea. A) Lado izquierdo, cara externa. Ba: basihial. B) Vistas ventral (1), dorsal (2) y lateral (3) del urohial. Cer: ceratohial; Epi: epihial; Hinf: hipohial inferior; Hsup: hipohial superior; Inte: interhial; R.B: radios branquiostegos. Escalas: 5 mm

Hyoid region of B. aurea. A) outer left side. B) ventral (1), dorsal (2) and lateral (3) views of urohyal. Ba: basihyal; Cer: ceratohyal; Epi: epihyal; Hinf: lower hypohyal inferior; Hsup: upper hypohyal; Inte: interhyal; R.B: branchiostegal rays. Scale bars: 5 mm 
A

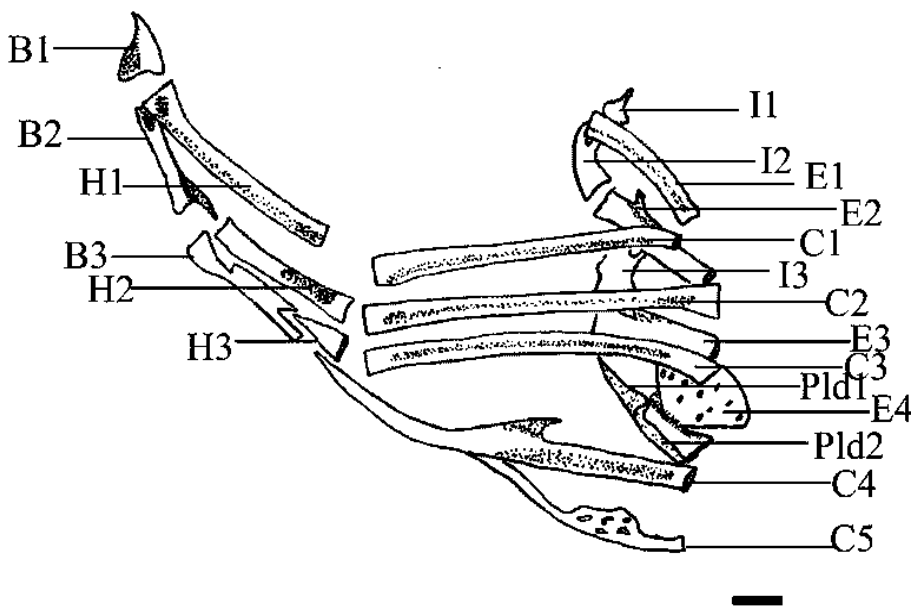

$\mathrm{B}$

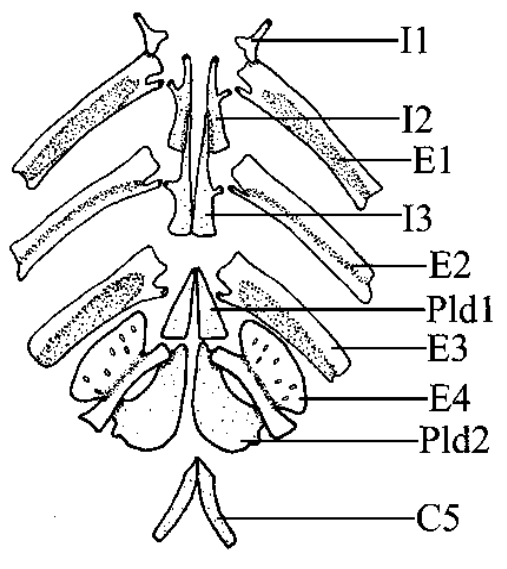

Figura 5

Arcos branquiales izquierdos de B. aurea. A: en vista lateral; B: región posterior en vista dorsal. B1, B2 y B3: basibranquiales; C1, C2, C3, C4 y C5: ceratobranquiales; E1, E2, E3 y E4: epibranquiales; H1, H2 y H3: hipobranquiales; I1, I2 y I3: infrafaringobranquiales; Pld1: primer par de placas dentarías; Pld2: segundo par de placas dentarias. Escalas: 5 mm

Left branchial arches of B. aurea. A: lateral view; B: posterior region in dorsal view. B1, B2 and B3: basibranchials; C1, C2, C3, C4 and C5: ceratobranchials; E1, E2, E3 and E4: epibranchials; H1, H2 and H3: hypobranchials; I1, I2 and I3: infrapharyngobranchials; Pld1: first pair of dental plates; Pld2: second pair of dental plates. Scale bars: $5 \mathrm{~mm}$

con los epibranquiales 2 (Fig. 5B). En la región posterior de los arcos branquiales, entre el $3^{\text {er }}$ infrafaringobranquial y el $4^{\circ}$ epibranquial, existen dos pares de placas dentarias óseas en posición vertical. Las primeras (Pld1) son alargadas y de forma triangular, encontrándose en la línea media a continuación del $3^{\text {er }}$ par de infrafaringobranquiales. Ventralmente continúan con el otro par de placas (Pld2) que son grandes y se encuentran apoyadas a los epibranquiales $4^{\circ}$. Las placas dentarias en los adultos carecen de dientes (Fig. 5B).

\section{Esqueleto axial postcraneal}

Esta región está constituida por la columna vertebral formada por las vértebras precaudales (9 a 11) y caudales (31 a 36), las costillas, huesos intermusculares y esqueleto caudal. Las costillas están presentes desde la $4^{a}$ vértebra precaudal hasta la vértebra 14-16 caudal, y tienen forma de varilla alargada (Fig. 6). 

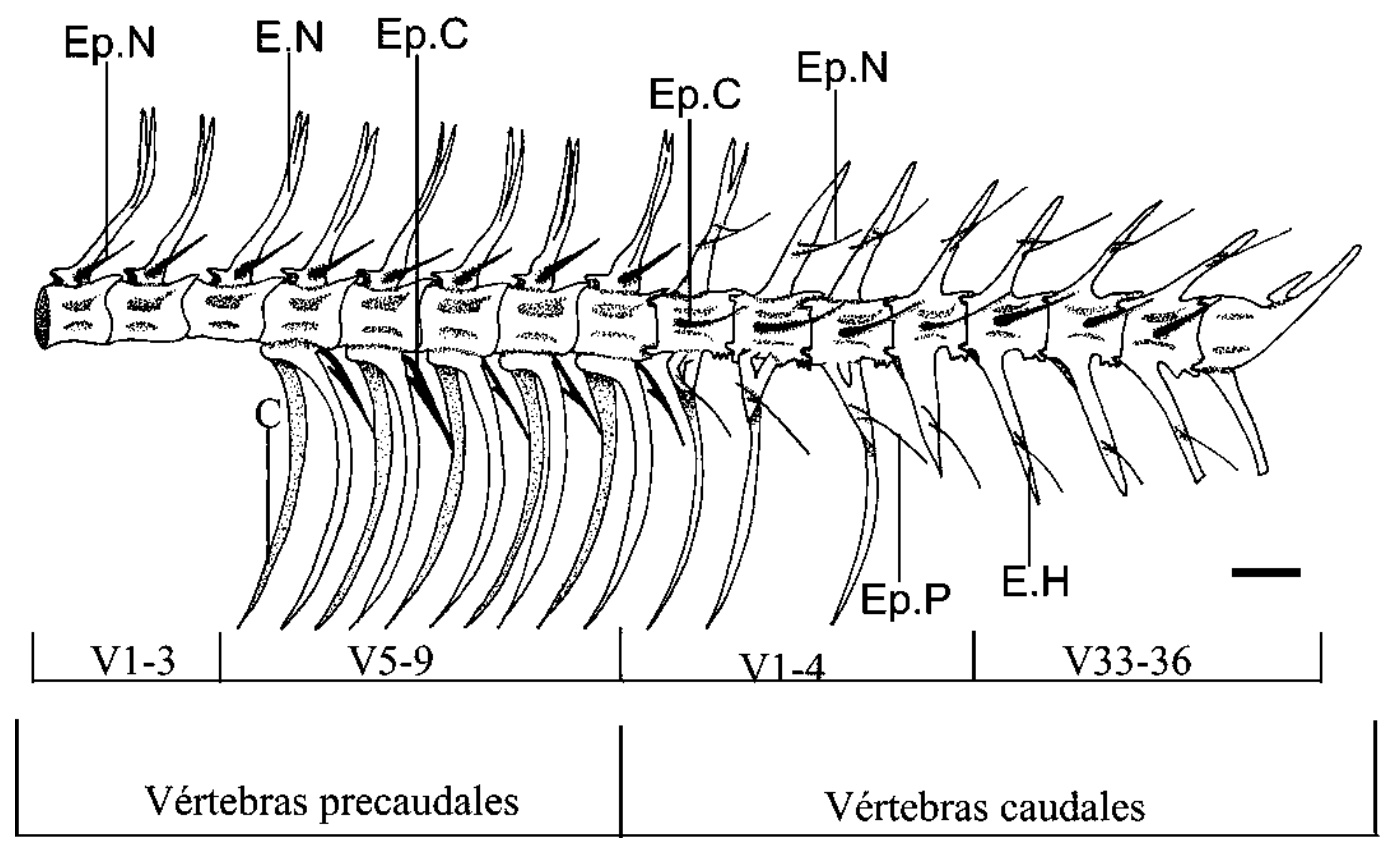

Figura 6

Esqueleto axial poscraneal de B. aurea. C: costilla; E.H: espina hemal; E.N: espina neural; Ep.C: epicentral; Ep.N: epineural; Ep.P: epipleural. Escala: 5 mm

Postcraneal axial skeleton of B. aurea. C: rib; E.H: haemal spine; E.N: neural spine; Ep.C: epicentral bone;

Ep.N: epineural bone; Ep.P: epipleural bone. Scale bar: 5 mm

Los huesos intermusculares se distinguen de las costillas porque se encuentran en los miosepta. Existen tres series de huesos intermusculares en Brevoortia: epineural (Ep.N), epicentral (Ep.C) y epipleural (Ep.P) (Patterson \& Johnson 1995). Los huesos epineurales se encuentran tanto en las vértebras precaudales como en las caudales, los epicentrales se encuentran tanto en las vértebras precaudales como caudales, y los epipleurales en las vértebras caudales (Fig. 6). La serie epicentral en los Clupeiformes presenta una característica única entre los teleósteos y es que están fusionados con las costillas anteriores (Patterson \& Johnson 1995). Los epicentrales se caracterizan por estar fusionados al centro vertebral de las vértebras caudales (Fig. 6). Los epipleurales en las vértebras precaudales, tienen su origen en la porción dorsal de las costillas pleurales. En las vértebras caudales, los epipleurales articulan con las espinas hemales (Fig. 6).

El esqueleto caudal está formado por un parhipural $(\mathrm{Ph})$, seis hipurales (Hp1-6), dos epurales (Ep1-2) y dos uroneurales (Un1-2). El parhipural (Ph) y el hipural 2
(Hp.2) se apoyan sobre el centrum preural 1 (PU1) + centrum ural 1 (U1). El centrum preural 1 es el último centrum que posee arco hemal. Los centra urales son los que se encuentran inmediatamente por detrás del centrum preural 1, el centrum ural 1 está fusionado con el centrum preural 1. Posterior al centrum ural 1 se encuentra el centrum ural 2 (U2) de pequeño tamaño sirviendo de soporte al hipural 3 (Hp.3). El primer hipural (Hp.1) queda a cierta distancia del centrum careciendo de articulación basal con el centrum ural 1. Los hipurales 4, 5 y 6 son gradualmente de menor tamaño. Existen dos uroneurales alargados y delgados. Los epurales (Ep) son huesos de pequeño tamaño, situados entre el extremo del urostilo y la espina neural (EN) (Fig. 7).

\section{Aletas dorsal y anal}

Las aletas están constituidas por 16 a 19 radios (Ra), los cuales articulan con un pequeño pterigióforo distal (Pt.D), constituido por dos mitades lentiformes y por los pterigióforos proximales (Pt.P), que están alargados dorsoventralmente. Los primeros tres pterigióforos 
proximales en la aleta dorsal son prominentes y anchos (Fig. 8). En la aleta anal el primer pterigióforo proximal se caracteriza por ser delgado y por presentar una ligera curvatura en el extremo dorsal (Fig. 9). Los primeros dos radios de la aleta anal son pequeños y articulados al primer pterigióforo distal (Fig. 9).

\section{Esqueleto apendicular}

Está constituido por las cinturas escapular y pélvica y sus respectivas aletas, pectoral y pélvica.
La cintura escapular está constituida por el postemporal (Post), cleitrum (Cl), coracoides (Co), mesocoracoides (Mco), la escápula (Esc), tres series de radiales pectorales (R.p, R.c y R.d) y supracleitrum (Sup) y dos poscleitra (Post.s y Post.i) (Fig. 10). El postemporal es un hueso alargado que está dividido dorsalmente en tres procesos. Este hueso une la cintura escapular al cráneo por medio de los supracleitra (Fig. 10).

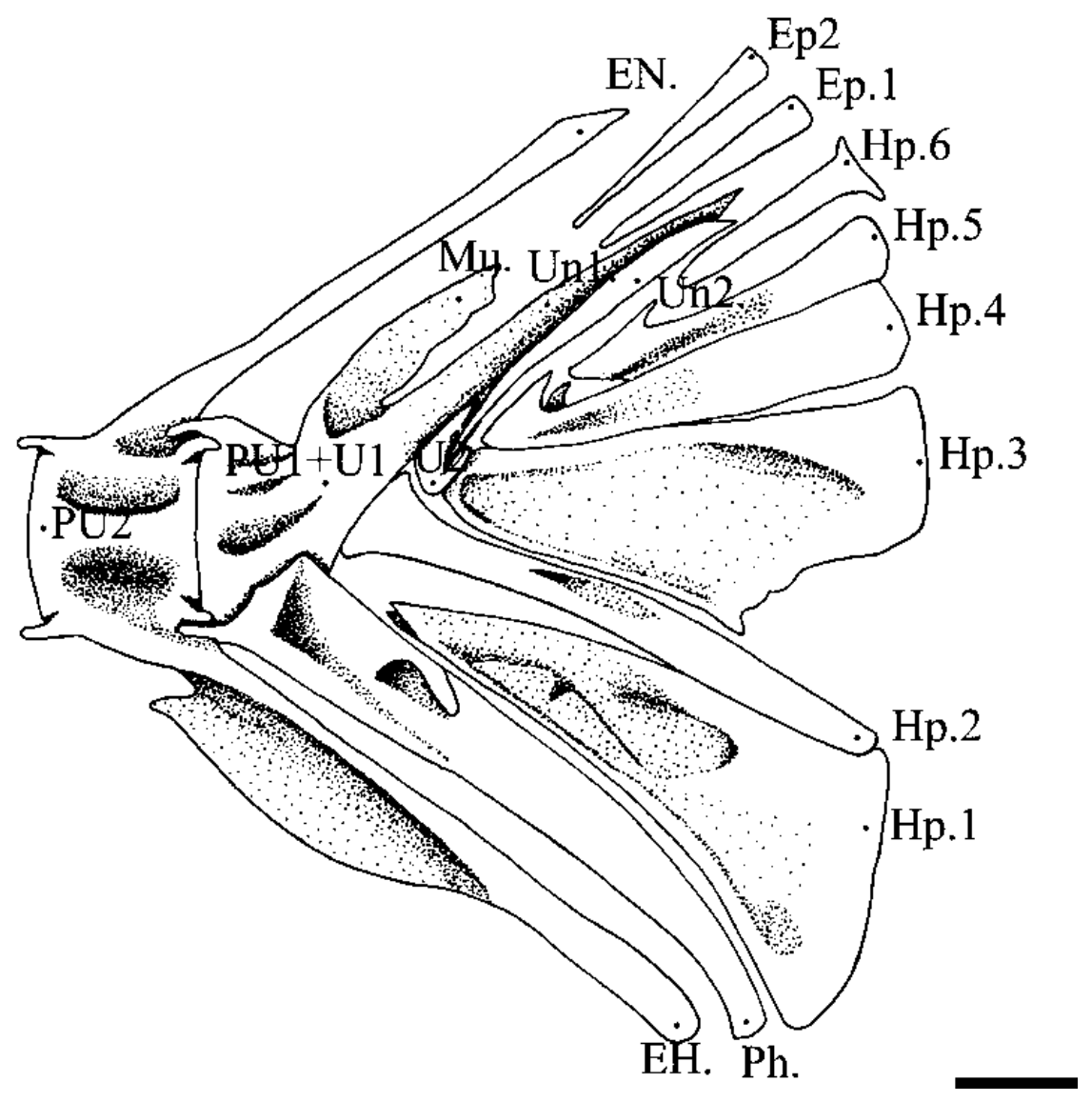

Figura 7

Complejo hipural de B. aurea. EH.: espina hemal; EN.: espina neural; Ep: epural; Hp: hipural; Mu: membrana ósea del primer uroneural; Ph: parhipural; PU1+U1: centrum preural 1 + ural 1; PU2: centrum preural 2; U2: centrum ural 2; Un: uroneurales. Escala: 5 mm

Hypural complex of B. aurea. EH.: haemal spine; EN.: neural spine; Ep: epural; Hp: hypural; Mu: bony membrane of first uroneural; Ph: parahypural; PU1+U1: preural centrum 1 + ural 1; PU2: preural centrum 2; U2: ural centrum 2; Un: uroneurales. Scale bar: 5 mm 


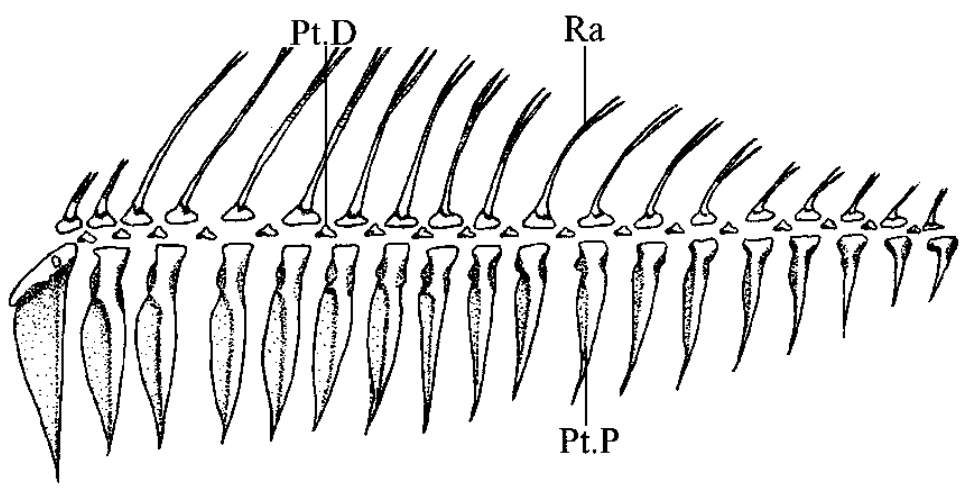

Figura 8

Aleta dorsal de B. aurea. Pt.D: pterigióforos distales; Pt.P: pterigióforos proximales; Ra: radios. Escala: 5 mm Dorsal fin of B. aurea. Pt.D: distal pterygiophores; Pt.P: proximal pterygiophores; Ra: rays. Scale bar: $5 \mathrm{~mm}$

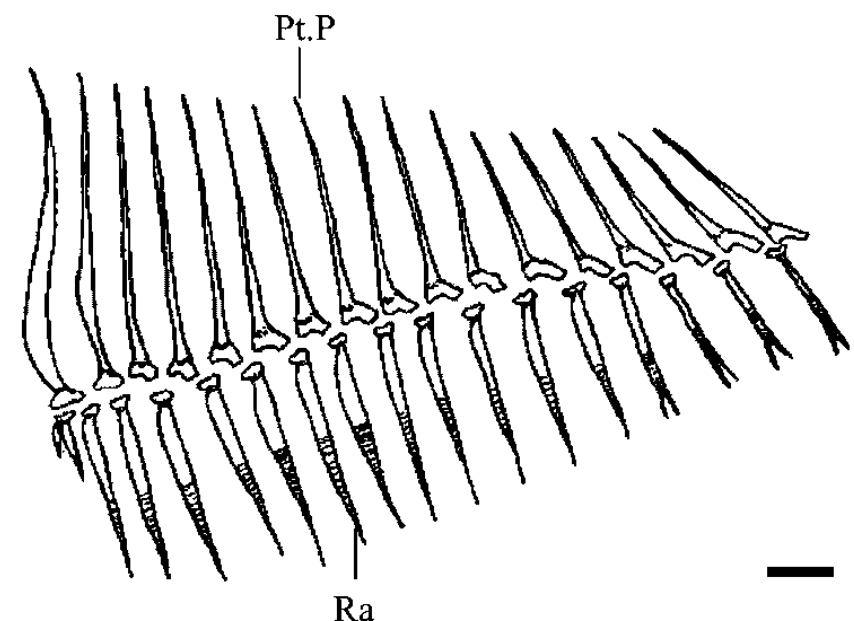

Figura 9

Aleta anal de B. aurea. Pt.P: pterigióforos proximales; Ra: radios. Escala: 5 mm Anal fin of B. aurea. Pt.P: proximal pterygiophores; Ra: rays. Scale bar: 5 mm 
A

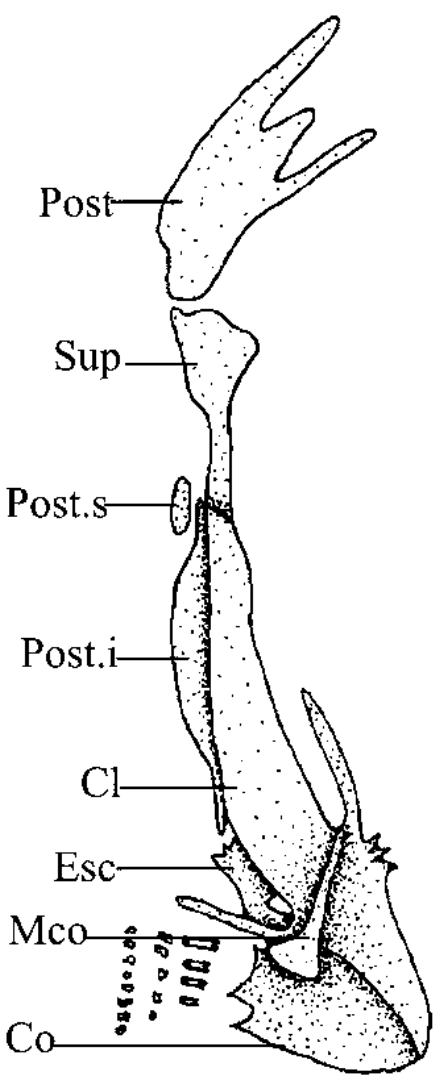

B

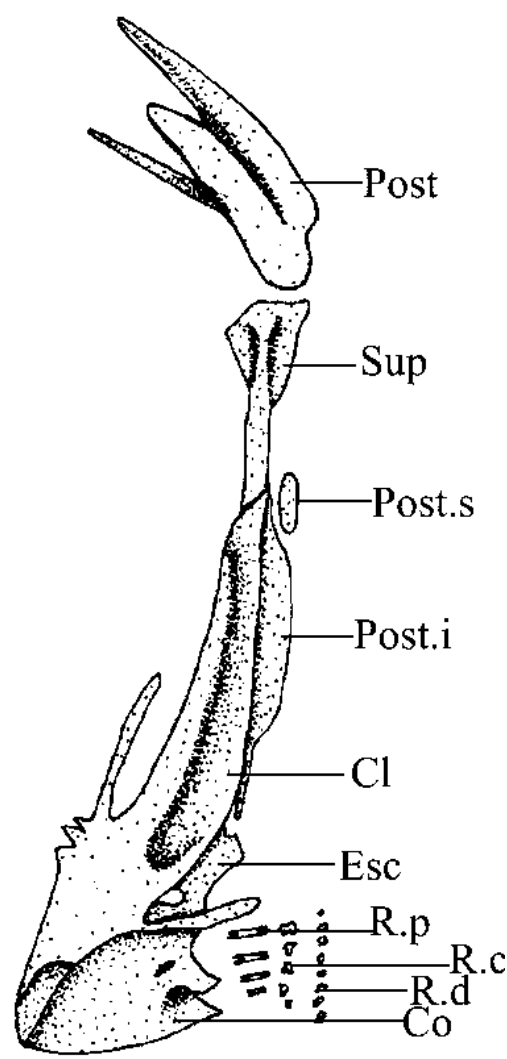

Figura 10

Cintura escapular y aleta pectoral (lado izquierdo) de B. aurea. A: cara interna y B: cara externa. Cl: cleitrum; Co: coracoides; Esc: escápula; Mco: mesocoracoides; Post: postemporal; Post.s: postcleitrum superior; Post.i: postcleitrum inferior; R.c: radiales centrales; R.d: radiales distales; R.p: radiales proximales; Sup: supracleitrum. Escala: 5 mm

Pectoral girdle and pectoral fin (left side) of B. aurea. A: inner view B: outer view. Cl: cleithrum; Co: coracoid; Esc: scapula; Mco: mesocoracoid; Post: post-temporal; Post.s: postcleithrum superior; Post. i: postcleithrum inferior; R.c: central radials; R.d: distal radials; R.p: proximal radials; Sup: supracleithrum. Scale bar: 5 mm

El cleitrum presenta forma sigmoidea, con superficies de articulación. La escápula tiene forma rectangular y presenta un amplio foramen en su extremo anterior. Los huesos coracoides, mesocoracoides, supracleitrum y poscleitra son laminares. El coracoides se caracteriza por presentar un proceso en la zona central de la cara externa, que articula con la escápula. El mesocoracoides tiene forma de varilla y el supracleitrum es un hueso alargado que se estrecha ventralmente. El poscleitrum superior es de pequeño tamaño y ovalado, mientras que el poscleitrum inferior está alargado en el eje vertical, termina en punta en el extremo ventral (Fig. 10) y presenta una concavidad en el extremo dorsal que toma contacto con el poscleitrum superior. Hay 3 hileras de pequeños radiales (proximal, central y distal) de forma irregular (Fig. 10).

La cintura pélvica está formada por un par de basipterigios (Bas), de forma triangular y por dos o tres pequeños radiales pélvicos (Rad) (Fig. 11). 


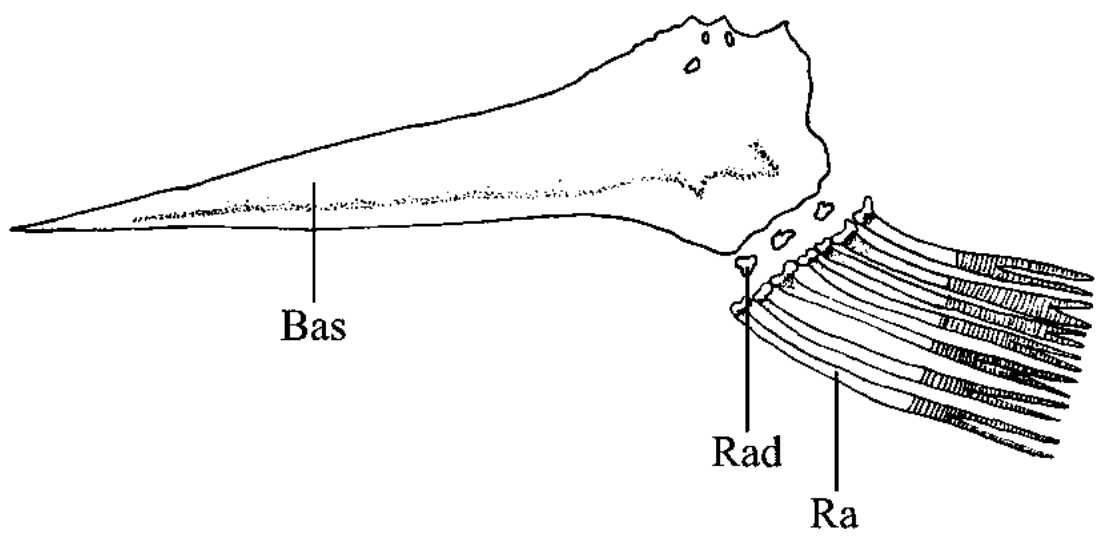

Figura 11

Cintura y aleta pélvica (lado izquierdo) de B. aurea. Cara externa, vista lateral. Bas: basipterigio; Ra: radios; Rad: radiales. Escala: 5 mm

Pelvic girdle and pelvic fin (left side) of B. aurea. Outer side, lateral view. Bas: basipterygium; Rad: radials; Ra: rays. Scale bar: 5 mm

\section{Discusión}

El cráneo de los Clupeomorpha presenta tres características notables y constantes: las fosas temporales, las fosas pre-epióticas y las fosas o fenestras auditivas (Cervigón 1980). En Brevoortia aurea estas tres características se presentan invariablemente en todos los especímenes analizados. Sin embargo, en otras especies de clupeidos, los tamaños relativos de estas aberturas craneales muestran una considerable variación interespecífica (Greenwood et al. 1966), pudiendo en algunos géneros, una o más aberturas, estar ausentes. Por ejemplo, en Clupea finta Cuvier las aberturas son poco conspícuas y la ventana o fenestra auditiva puede incluso estar ausente (Gregory 1933). El prévomer en $B$. aurea no lleva dientes. En otras especies de clupeidos, en cambio, puede adosarse una placa dentaria, como en Jenkinsia lamprotaenia (Gosse) (Cervigón \& Velázquez 1978). Las bullas óticas en $B$. aurea son ovaladas y de gran tamaño, y se desarrollan en los huesos proóticos. Son bien prominentes también en $B$. tyrannus (Latrobe), aunque prácticamente inexistentes en Ethmalosa fimbriata (Bowdich), un clupeido estrechamente emparentado con Brevoortia (Monod 1961). La fusión de los huesos extraescapulares y parietales es considerada como una sinapomorfía del clado Clupeomorpha + Ostariophysi (Lecointre \& Nelson 1996). Sin ambargo, en B. aurea dichos huesos no se observaron fusionados. Tampoco fueron observados en Jenkinsia lamprotaenia, especie en que el extraescapular se une dorsalmente al parietal por tejido conectivo (Cervigón \& Velázquez 1978).

La forma y el tamaño de la cabeza y las mandíbulas entre los peces clupeomorfos es una característica variable, aunque existe una tendencia hacia la reducción de dientes como una adaptación a la alimentación micrófaga (Whitehead 1985). La saraca, B. aurea carece de dientes en las mandíbulas en etapas juvenil y adulta. Sin embargo, han sido detectados pequeños dientes en estadios larvarios que luego desaparecen a partir de los 30 mm de longitud total (Giangiobbe \& Sánchez 1993). En las primeras etapas de vida de $B$. tyrannus se observa una dentición conspicua, consecuencia de una dieta zooplanctófaga, que luego cambia hacia una alimentación planctófaga con pérdida de los dientes (June \& Carlson 1971). En otros clupeidos, en cambio, la presencia de dientes persiste hasta la etapa adulta, como en la subfamilia Chirocentrinae (Lecointre \& Nelson 1996) o en Pellona Valencienne (Whitehead 1973). La tendencia hacia la reducción de dientes en los clupeidos, asociada a una dieta micrófaga, va acompañada de un desarrollo de los órganos suprabranquiales que se asocian con los arcos branquiales cuarto y quinto (Greenwood et al.1966). En Brevoortia aurea, el cuarto par de epibranquiales junto al quinto arco branquial, constituyen el órgano o bolsa epibranquial. El órgano epibranquial es una estructura par situada por encima y detrás de los arcos branquiales. Consta de un canal o conducto, y una cavidad o lumen, con dos hileras de branquispinas. Su función está 
vinculada con el aparato digestivo (Sánchez 1989, Giangiobbe \& Sánchez 1993) y generalmente se considera que funciona como un reservorio temporal para la acumulación y concentración de alimento (Cervigón 1980). El órgano epibranquial también está bien desarrollado en $B$. tyrannus y Ethmalosa fimbriata (Monod 1961), sin embargo en especies de Alosa Linck y Clupea harengus Linnaeus los órganos epibranquiales están muy poco desarrollados 0 son vestigiales (Cervigón 1980).

Las características del urohial en $B$. aurea, con sus dos procesos anteriores bien definidos y sus procesos carenados o alares lateroposteriores, también están presentes en $B$. tyrannus, Ethmalosa fimbriata y en otros géneros de la familia Clupeidae (Monod 1961).

El esqueleto caudal de Brevoortia aurea corresponde al tipo denominado pleurostilar (Patterson 1968) en el que uno de los grandes uroneurales (en nuestro caso el uroneural 1) se fusiona con el centrum preural 1 y ural para constituir el pleurostilo (Monod 1968). Grande (1982) considera al pleurostilo como resultado de la fusión del uroneural 1 con el centrum preural 1. La fusión del hipural 2 con el centrum ural 1 es una sinapomorfía de los clupeomorfos (Lecointre \& Nelson 1996), aunque en el caso de B. aurea, estos huesos no se encuentran fusionados (Fig. 7). En otros clupeidos se ha observado que el segundo hipural está más o menos fusionado con el centrum ural 1, como en Jenkinsia spp. Jordan \& Evermann o incluso no hay fusión entre dichos elementos como ocurre en Spratelloides spp. Bleeker (Cervigón \& Velázquez 1978).

Las espinas hemales anteriores al centrum preural 1 se encuentran fusionadas en Brevoortia aurea (Figs. 6 y 7). Fujita (1990) demostró que la fusión de las espinas hemales anteriores al centrum preural 2 probablemente está presente en todos los clupeomorfos. La presencia de los huesos intermusculares epicentrales es ampliamente reconocida en todos los clupeomorfos (Patterson \& Johnson 1995) y fue observada en $B$. aurea.

Es peculiar la presencia de tres hileras de huesos radiales pectorales (proximal, central y distal) en Brevoortia aurea (Fig. 10). Según Cervigón (1980) la presencia de una hilera de radiales pectorales, además de la proximal en Clupeomorpha, no es citada en la literatura clásica. Entre los clupeidos, hay dos hileras de radiales pectorales en el género Lile Jordan \& Evermann (Gómez 1973) y tres en Jenkinsia (Cervigón \& Velázquez 1978).

\section{Agradecimientos}

Los autores desean expresar su agradecimiento a los Dres. Daniel Figueroa y María Berta Cousseau, por sus sugerencias y colaboración en los muestreos. Al Dr. Brian Dyer y al Prof. Ismael Kong por la dedicación y esmero en sus correcciones críticas y sugerencias que contribuyeron a mejorar sustancialmente este trabajo.

\section{Literatura citada}

Berry FH. 1964. Review and emendation of Family Clupeidae by S. Hildebrand. Copeia 1964: 720-730.

Carvalho-filho A. 1999. Peixes: costa brasileira $3^{\text {a }}$ ed., 320 pp. Melro Ltd., São Paulo, Brasil.

Cassia MC \& S García de la Rosa. 1993. Características diferenciales del desarrollo larval de Brevoortia aurea (Pisces, Clupeidae) en el Atlántico suroccidental. Frente Marítimo 14: 63-69.

Cervigón F. 1980. Ictiología marina. Vol. 1, 358 pp. Ed. Arte, Caracas, Venezuela.

Cervigón F \& E Velázquez. 1978. Revisión sistemática y estudio osteológico de las especies del género Jenkinsia de las costas de Venezuela (Osteichthyes: Clupeiformes). Cuadernos Oceanográficos Universidad de Oriente 7: 354.

Cousseau MB \& MA Denegri. 1997. Peces. En: Cousseau MB (ed). Peces, crustáceos y moluscos registrados en el sector del Atlántico suroccidental comprendido entre $34^{\circ} \mathrm{y}$ $55^{\circ} \mathrm{S}$, con indicación de las especies de interés pesquero. Pp 9-48. INIDEP, Informe Técnico 5.

Cousseau MB \& JM Díaz de Astarloa. 1993. El género Brevoortia en la costa Atlántica sudamericana. Frente Marítimo 14: 49-57.

Cousseau MB, H Nion, MA Denegri \& S Olivera. 1998. Lista de peces de la Zona Común de Pesca ArgentinoUruguaya. Frente Marítimo 17: 123-151.

De Buen F. 1950. El Mar de Solís y su fauna de peces $\left(2^{\mathrm{a}}\right.$ parte). Servicio de Oceanografía y Pesca del Uruguay. Publicación Científica 2: 46-144.

Díaz de Astarloa JM. 1994. Las especies del género Paralichthys (Pisces, Paralichthyidae) del mar argentino. Morfología y sistemática. Tesis Doctoral. Universidad Nacional de Mar del Plata, Argentina, 194 pp.

Figueiredo JL \& NA Menezes. 1978. Manual de peixes marinhos do sudeste do Brasil. II. Telesostei (1), 110 pp. Museu de Zoologia, Universidade de São Paulo.

Fujita K. 1990. The caudal skeleton of Teleostean fishes, 914 pp. Tokai University Press, Tokyo. 
Giangiobbe A \& F Sánchez. 1993. Alimentación de la saraca (Brevoortia aurea). Frente Marítimo 14: 71-80.

Gómez A. 1973. Estudio osteológico de Lile piquitinga Schreiner y Miranda Ribeiro (Pisces: Clupeidae). Tesis de Maestría, Instituto Oceanográfico, Universidad de Oriente, Venezuela, 46 pp.

Greenwood PH, DE Rosen, SH Weitzman \& GS Myers. 1966. Phyletic studies of Teleostean fishes, with a provisional classification of living forms. Bulletin of the American Museum of Natural History 131: 339-456.

Gregory WK. 1933. Fish skulls. A study of the evolution of natural mechanisms. Transactions of the American Philosophical Society 23: 75-481.

Grande L. 1982. A revision of the fossil genus Knightia, with a description of a new genus from the Green River Formation (Telesotei, Clupeidae). American Museum Novitates 2731: 1-22.

Hildebrand SF. 1948. A review of the American menhaden, genus Brevoortia, with a description of a new species. Smithsonian Miscellaneous Collections 107: 1-38.

Hormaechea A. 1983. Determinación de los caracteres morfométricos y merísticos de la saraca, Brevoortia spp. Tesis de grado. Universidad Nacional de Mar del Plata, Argentina. 37 pp.

Lecointre G \& G Nelson. 1996. Clupeomorpha, sister-group of Ostariophysi. En: Stiassny MLJ, LR Parenti \& GD Johnson (eds). Interrelationships of fishes, pp. 193-207. Academic Press, San Diego, California.

June F \& F Carlson. 1971. Food of young Atlantic menhaden, Brevoortia tyrannus in relation to metamorphosis. Fishery Bulletin 68: 493-512.

Kawamura K \& K Hosoya. 1991. A modified double staining technique for making a transparent fish-skeletal specimen. Bulletin of the National Research Institute of Aquaculture 20: 11-18.

Mayden RL \& EO Wiley. 1984. A method of preparing disarticulated skeletons of small fishes. Copeia 1984: 230232.

Menezes NA, PA Buckup, JL de Figueiredo \& RL de Moura. 2003. Catálogo das espécies de peixes marinhos do Brasil. Museu de Zoologia da Universidade de São Paulo, Brasil.

Menni RC, RA Ringuelet \& RH Arámburu. 1984. Peces marinos de la Argentina y Uruguay. Reseña histórica. Clave de familias, géneros y especies. Catálogo crítico, 359 pp. Hemisferio Sur S.A., Buenos Aires.
Monod T. 1961. Brevoortia Gill 1861 et Ethmalosa Regan 1917. Bulletin de l' Institute Fondam Africa Noire 23: 506-547.

Monod T. 1968. Le complex urophore des poissons téleóstéens. Mémoires de l’Institute Fondam Africa Noire 81: 1-705.

Patterson C. 1968. The caudal skeleton on lower liassic pholidophorid fishes. Bulletin of the British Museum (Natural History) Geology 16: 203-239.

Patterson C \& GD Johnson. 1995. The intermuscular bones and ligaments of teleostean fishes. Smithsonian Contributions to Zoology 559: 1-83.

Reintjes JW. 1969. Synopsis of biological data on the Atlantic menhaden, Brevoortia tyrannus. United States Wildlife Service Circular 320, 30 pp.

Ringuelet RA. \& RH Arámburu. 1960. Peces marinos de la República Argentina. Clave de familias y géneros y catálogo crítico abreviado. Agro 5: 1-141.

Ringuelet RA, RH Aramburu \& AA de Aramburu. 1967. Los peces argentinos de agua dulce. Comisión de Investigación Científica, 602 pp. Provincia de Buenos Aires. (CIC), Buenos Aires.

Rojo AL. 1988. Diccionario enciclopédico de anatomía de peces. Monografía del Instituto Español de Oceanografía 3: $1-566$.

Sánchez F. 1989. Características morfológicas del aparato digestivo y espectro trófico de la saraca (Brevoortia aurea, Clupeiformes, Pisces). Physis (Buenos Aires), Sección A 47: 21-33.

Wasserssug RJ. 1976. A procedure for differential staining of cartilage and bone in whole formalin-fixed vertebrates. Stain Technology 51: 131-134.

Whitehead PJP. 1973. The clupeoid fishes of the Guainas. Bulletin of the British Museum (Natural History) Zoology, supplement 5: 1-227.

Whitehead PJP. 1985. FAO species catalogue. Clupeoid fishes of the world (Suborder Clupeoidei). An annotated and illustrated catalogue of the herrings, sardines, pilchards, sprats, schads, anchovies and wolf-herrings. Part I. Chirocentridae, Clupeidae and Pristigasteridae. FAO Fisheries Synopsis 125, V. 7, Pt I, 303 pp. 